University of Nebraska - Lincoln

DigitalCommons@University of Nebraska - Lincoln

7-2011

Networked Expertise in the Era of Many-to-many Communication: On Wikipedia and Invention

\author{
Damien S. Pfister \\ University of Nebraska-Lincoln, dpfister2@unl.edu
}

Follow this and additional works at: https://digitalcommons.unl.edu/commstudiespapers

Part of the Communication Technology and New Media Commons, and the Speech and Rhetorical

Studies Commons

Pfister, Damien S., "Networked Expertise in the Era of Many-to-many Communication: On Wikipedia and Invention" (2011). Papers in Communication Studies. 10.

https://digitalcommons.unl.edu/commstudiespapers/10

This Article is brought to you for free and open access by the Communication Studies, Department of at DigitalCommons@University of Nebraska - Lincoln. It has been accepted for inclusion in Papers in Communication Studies by an authorized administrator of DigitalCommons@University of Nebraska - Lincoln. 


\title{
Networked Expertise in the Era of Many-to-Many Communication: On Wikipedia and Invention
}

\section{Damien Smith Pfister}

Department of Communication Studies, University of Nebraska-Lincoln, 439 Oldfather Hall, Lincoln, NE 68588, USA; email dpfister2@unl.edu

\begin{abstract}
This essay extends the observations made in E. Johanna Hartelius' The Rhetoric of Expertise about the nature of expertise in digital contexts. I argue that digital media introduce a scale of communication - many-to-many - that reshapes how the invention of knowledge occurs. By examining how knowledge production on Wikipedia occurs, I illustrate how many-to-many communication introduces a new model of "participatory expertise." This model of participatory expertise challenges traditional information routines by elevating procedural expertise over subject matter expertise and opening up knowledge production to the many. Additionally, by hosting multiperspectival conversations on Wikipedia, the participatory model of expertise introduces epistemic turbulence into traditionally tranquil encyclopedia culture.
\end{abstract}

Keywords: many-to-many communication, invention, expertise, multiperspectivalism, epistemic turbulence

\section{Introduction}

Is there a keyword of digital culture that inspires more polarized reactions than Wikipedia? At one pole are those that see in Wikipedia a hopeful glimpse of the future: communication networks that navigate around old power relations in order to arrive at consensus about human knowledge. At the opposite pole are the critics - advocates of traditional encyclopedias, skeptics of lay participation in expert fields, and, yes, many teachers - who are suspicious of how Wikipedia disrupts traditional routines of knowledge production ${ }^{1}$ and circulation. Both defenders and critics, however, agree that Wikipedia is a consequential node of influence in emer- 
gent digital communication networks. In fact, it is perfectly sensical to spoof the idea of social constructionism with the term "Wikipedia constructionism." Search engines have a crucial role in shaping how we now perceive the world and the top hit for almost any search is often a Wikipedia page (Halavais 2009). Wikipedia is thus a key example of how the new contexts of many-to-many communication afforded by the internet alter cultural practices. This essay specifically identifies how the many-to-many communication occurring on Wikipedia challenges traditional models of expertise by disrupting established information routines and cultivating multiperspectivalism.

Given the significance of Wikipedia, critical inquiry into how it shapes contemporary public culture is crucially important. An extension of the polarized discussion that dominates popular dissection of Wikipedia is less helpful than a more pragmatic and nuanced assessment in situating this digital phenomenon historically and theoretically (Carey 2005). E. Johanna Hartelius' chapter "Wikipedia: The rhetoric of informational expertise," in The Rhetoric of Expertise (Hartelius 2011), sensitively analyzes encyclopedias old and new in order to theorize how each conceives of expertise. As an historical contribution to our understanding of Wikipedia, Hartelius' chapter explores the roots of encyclopedia culture in the modern impulse to gather, systematize, and distribute knowledge. This modern inclination to organize knowledge, an outgrowth of the Enlightenment, produced particular forms of expertise that revolve around professional training, accreditation, and academic peer review. These forms of modern expertise provide a useful contrast to emergent forms of networked expertise. Wikipedia is one such model of networked expertise where information flows from multiple, often peripheral, nodal points toward a central, aggregating node.

Theoretically, Hartelius identifies not the conditions that make Wikipedia superior to the Encyclopaedia Britannica, or vice versa, but the tensions that emerge from the clash of modern expertise with networked expertise. By juxtaposing discourse published on Wikipedia with statements from its critics in other venues, she identifies how each side in this debate over authority relies on different - which is to say contingent-models of expertise. The communicative self-reflexivity of Wikipedia is contrasted with the scholarly authority of the Encyclopaedia Britannica in a series of subsidiary tensions: quantity versus quality, passion versus disinterest, timeliness versus timelessness, dialogue versus monologue, consensual decision-making versus editorial judgment, practical wisdom versus theoretical knowledge, and, perhaps most intriguing, network trust versus expert trust. As Hartelius makes clear, both Wikipedia and more traditional encyclopedias construct their own criteria of expertise. However, her comparison of Wikipedia and the Encyclopaedia Britannica does not overemphasize their differences. Each encyclopedia project shares similarities in as much as they both have preferences for the anonymity of the contributor, belief in neutrality and objectivity, conviction in the value of compiling human knowledge, and an articulated commitment to the public good. 
If there is a limitation to Hartelius' analysis, it is that she does not take the opportunity to more extensively theorize the different norms of expertise in these two encyclopedias as indicative of a paradigmatic shift in how knowledge is created, negotiated, and circulated in internetworked societies. In this essay, I make a case for how the rise of many-to-many networked communication challenges the modernist regime of expertise. I then explore how many-to-many communication reshapes the relationship between invention and expertise. Although I am arguing that networked expertise is a considerable departure from modern expertise, I hope to do so in a way that does not uncritically fetishize Wikipedia. However, it is possible that in characterizing Wikipedia as a sea change in expertise, I am overstating the case for how digital networks transform communication. With that caveat, let me state a hope that the pairing of this essay with Hartelius' chapter will perform that essential function of good companionships: providing balance for each other.

\section{Models of Communication/Models of Expertise}

Internetworked technologies offer the first credible medium for many-to-many communication, where interlocutors can sustain large-scale, interlinked, synchronous and asynchronous contact. Many-to-many communication continues a historical trend of new information technologies increasing the scale of communicative association that both accommodates and facilitates ever more complex societies. Wikipedia is a signature example of many-to-many communication. Many articles are the product of hundreds or thousands of different contributors, who use a variety of ways to negotiate differences of opinion in real time and over time.

If historical trends hold, this new scale of communication will have appreciable impact: "Since the first expansive one-to-one communications medium (language) helped distinguish humans from apes and the first one-to-many communications medium helped make Europe the first modern society," ask James Dewar and Peng Hwa Ang (2007, 366-67), "what impact might the latest fundamental change in that chain (many-to-many) have?" One-to-one communication captures the interpersonal interactions usually mediated by the voice and supportive of smaller-scale societies. Oneto-many is the broadcast model perfected by the mass media and associated most closely with modern society since the print revolution (although as imperial proclamations illustrated, writing could function as a mass medium). Although overlooked by Dewar and Ang, many-to-one communication is also a link in this chain - as when citizens write letters or emails to public officials. The internet, notably, hosts manyto-many communication but also is a medium that, unlike any other, supports the earlier communication models of one-to-one, one-to-many, and many-to-one (Stalder 2006, 181). This flexibility certainly does not mean that many-to-many communication has supplanted prior modes, as the continued prevalence of face-to-face interpersonal communication demonstrates. Indeed, rather than conceptualizing many-tomany contact as the pinnacle of human communicative achievement or the inevitable 
telos of technological development, these different modes of interaction are synchronous and overlapping in contemporary communication ecosystems.

These four models of communication can be read as models of expertise. One-toone communication lends itself to a "counselor model" of expertise like that practiced by Socrates or an aide to the throne. A counselor may claim special knowledge from individual experience, collective narratives and myths, or even fortune- telling; they may also aim to draw out dormant knowledge in the counseled through questioning. In the latter case, the counselor and counseled co-create knowledge through dialectical inquiry. This model is often found in quotidian contexts, like when one asks a friend or parent for advice. In this model of expertise, a learned person imparts wisdom to one seeking it through intimate conversation. Many-to-one communication lends itself to an "advisory model" of expertise. The US Presidential cabinet relies on an advisory model. In this model, expert advice from multiple perspectives is funneled to the "decider." One-to-many communication supports a "broadcast model" of expertise. Public health advice and the nightly network news fall into this model, because the message comes from a single point source and is distributed to many people. The Encyclopaedia Britannica essentially relies on the one-to-many model. Even though a few peers and editors help the "one" author along, thus suggesting that behind every one is a many, the same standardized set of encyclopedias is circulated to many recipients.

In each instance, the model of communication and the dominant medium of communication do not just incidentally coincide; the cultural use of the medium actually shapes the model of expertise. The familiar face-to-face settings and simpler problems of oral societies invited dialogue as a problem-solving technique. Complex and sprawling modern societies require more sophisticated ways of acquiring "inputs" to aid organizational decision-making and circulate expert advice to citizens. As Elizabeth Eisenstein (1979) notes, the printed word better allows the gradual accumulation of knowledge, as one text becomes the basis upon which new knowledge is invented. To extend a well-worn metaphor, standing on the shoulders of those who came before gave modern experts a higher vantage point from which to broadcast their findings and insights. This broadcast model of expertise was, if nothing else, an efficient way of communicating information to masses of people before the advent of digital technologies.

Many-to-many communication, enabled by the new technologies that allow digital mediation, generates novel inventional practices through a "participatory model" of expertise. Networked environments did not create a participatory model of expertise out of thin air - the classical Athenian agora and the European bourgeois public sphere were historical efforts to mobilize participation by the many as well. These earlier media cultures, however, were limited by social codes that restricted participation to an elite and by technology that stunted efficacious many-to-many communication. Since the internet draws in more participation by the demos, at least compared with prior media, it has regularly been identified as a democracy-enhancing technology (Johnson 2003). To say that Wikipedia is an exercise in democratic knowl- 
edge production is not to celebrate it as merely popular (or worse, to praise it uncritically), but to acknowledge it as a messy, deliberative process that attempts to forge enough agreement in the content of each article that all parties accept the current version as legitimate.

The import of many-to-many communication is apparent when comparing Wikipedia with other encyclopedias. Since Wikipedia opens knowledge production to the many, the scale of the enterprise affords a more encyclopedic experience. Encyclopaedia Britannica, like any print product, is limited by the page space it can dedicate to any particular entry and the time that its paid staff can dedicate to the project. This is the deep irony: as long as the traditional ideal of the encyclopedia as a "circle of all human knowledge" remains, print encyclopedias cannot be considered encyclopedic. Wikipedia might not circle up all human knowledge, but it certainly encompasses more than the Britannica. For Wikipedia, space is not a real constraint because data storage is cheap, and time spent working on the site is distributed across millions of volunteers (even though many or most edits are done by a relatively small cohort). For the sake of developing a more comprehensive reference source, this is probably a positive development: the sheer complexity and differentiation of the networked world defies the ability of a traditional paper encyclopedia to adequately represent it. The ability of many-to-many communication to produce a broader range of knowledge can be found in a comparison between the English version of Wikipedia, with over 3.5 million articles, and the Encyclopaedia Britannica, with just over 65,000 articles. Importantly, Wikipedia's broader array of articles has consistently been shown to match or approximate, and in some cases exceed, the reliability of traditional information sources (for details of studies and surveys confirming and questioning the accuracy of the project, see Wikipedia n.d. b).

\section{Practices of Invention in Many-to-many Networked Contexts}

Wikipedia, as an example of a participatory model of networked expertise, is a representative anecdote for the kinds of collaborative invention that digital media afford. My focus on invention departs a bit from Hartelius' primary distinction between the Encyclopaedia Britannica and Wikipedia. Hartelius argues that the construction of expertise on these two sites occurs dispositionally, which is to say that expertise emerges in how the information becomes arranged, or, to be more precise, in how that information is always in the process of becoming arranged. "To be an expert in disposition," Hartelius explains, "is to manage content analytically and deliberately" (2011, 138). The Encyclopedia Britannica manages content through layers of editorial control and (credentialed) peer feedback, resulting in a highly organized, fixed, linear text. Wikipedia, alternatively, can be edited by anyone. Indeed, contributors to Wikipedia are, in an egalitarian spirit, all called "editors." Each edit is tracked, which produces an "edit history," a publicly accessible record of all the changes that have been made to a particular entry. Often, the edit history features forum-style conversations 
and disputes. While most entries are organized systematically, the arrangement of information organically changes over time as users update and extend an article. And, of course, hypertext links interrupt the familiar linearity of print. These dispositional distinctions are crucial in marking the differences between the two encyclopedias.

While these differentiations are indeed important markers, privileging the first rhetorical canon of invention in my analysis rather than the second of disposition underlines how many-to-many communication represents a more fundamental shift in the way knowledge production itself occurs. It is important not to overdraw this delineation, for invention and disposition are mutually implicative. Indeed, by noting that Wikipedia's disposition demonstrates how "knowledge is a process of collaborative invention rather than the property of a single person," Hartelius illustrates how these two canons interact $(2011,147)$. However, the inventive practices of Wikipedia deserve more sustained attention. Such a focus yields two ways in which expertise is transformed through networked media; first, by destabilizing familiar information routines; and, second, by offering a site for multiperspectival exchange. Both points are intimately tied to how knowledge is collaboratively invented in networked societies.

First, Wikipedia destabilizes traditional information routines. New patterns of information flow enabled by digital media technology have recalibrated traditional expertise by relocating the rhetorical power of expertise from command of subject matter to procedural adroitness with information, and by expanding who contributes to subject matter expertise from the one to the many. On the first point: to a certain extent, modern expertise is simply based on access to and control of information. There is an insider/outsider dynamic to traditional models of expertise-some people have it, and some have not, and the have-nots are necessarily reliant on the haves. The history of expertise is bound up in this dynamic. The priests of the ancient Greeks were the only ones who could go inside the temples, leaving worshipers on the periphery. Similarly, the early Christian Church preserved sole interpretive authority over the Bible. The genesis of the term "expert" illuminates how established information routines maintain epistemic hegemony. As Steve Fuller notes, the word "expert," a contraction of the French "experienced," emerged in the context of court trials where the authenticity of handwriting was at stake $(2006,342)$. These handwriting experts were gifted at pattern recognition. Their successful track record of forgery detection gave them an epistemic authority not extended to non-experts: they were not expected to exhibit their reasoning publicly, nor could they be challenged by anyone but a fellow handwriting expert. Having become a priesthood of experts, they were hermetically sealed off from public criticism. Such an absolutely deferential relationship between experts and laypeople hardly remains. While networked societies are still reliant-maybe even more so than modern societies - on experts, their authority is no longer unquestioned. Doctors face patients armed with sheaves of WebMD print outs, scientific experts face scrutiny by legions of bloggers, and political pundits have lost their previously sacrosanct status to a host of online commentators. An expert's opinion might still have more swing 
than a layperson's, but it can no longer resist being drawn into a conversational circuit with so-called non-experts. Internetworked technologies draw in participation from the periphery and in some cases are outright removing traditional experts from their privileged role as intermediaries in public conversation.

Is expertise in a network society, then, little more than what Peter Walsh (2003) refers to as a "withered paradigm?" Perhaps withered, but certainly not wrecked - after all, many if not most Wikipedia articles cite evidence from traditional, credentialed experts, and many Wikipedia editors are traditional, bona fide experts. Critics of Wikipedia like Lawrence Sanger $(2009,61)$ point to this eventual "regress of credibility" that underpins many Wikipedia articles: in the end, traditional experts are invoked to decide matters. This focus on the role of experts risks missing how projects like Wikipedia function to reshape expertise. Some purchase on the difference between experts and expertise can be located in Hartelius' deft distinction between a subject matter expert and a procedural expert. Subject matter experts have readily recalled knowledge that can be employed with a level of execution greater than the non-expert. Recollection is the key term here, suggesting that expertise is traditionally tied to memory (Brandt, Copper, and Dewhurst 2005). An informational expert is not, Hartelius explains, like being "an expert on caterpillars or Shakespearean sonnets" $(2011,138)$. Rather, informational experts are experts on procedures: search, credibility assessment, synthesis, and deliberation are all processes that allow one to acquire subject matter expertise. With the internet, the historical power of subject matter expertise is eroded: the archival nature of the Web means that "what" and "how to" information is readily available. Recollection is simply less important with a smartphone in your pocket. Need to know something about caterpillars? There is a Wikipedia article on that. Curious about what a Shakespeare sonnet sounds like in period dialect? Check You-Tube. Want to know how to put up drywall, or make the perfect pasta? The instructions are a search away, and increasingly, as the advertising slogan goes, "there's an app for that." In digitally networked environments where many-tomany communication populates databases on various subjects, it is not as important to know about caterpillars or Shakespeare, it is important to know how to find information about caterpillars or Shakespeare.

Overplaying the decline in subject matter expertise would be a mistake-a doctor is still probably preferable to a neighbor for surgery, despite the fact your neighbor can view videos of surgery online. In fact, the science and technology controversies that populate contemporary life in networked societies - genetic modification, nanotechnology, and green energy, for example-are relatively new areas of research that will sustain genuinely new fields of subject matter expertise. Nevertheless, emerging in densely networked environments is a movement of expertise away from blunt forms of "credentialed" epistemic authority and back toward experience. Although execution, not recollection, now identifies a real subject- matter expert, knowledge of how to execute well can also be learned through the archives of the internet (although I would still suggest a wee bit of practice before reaching for the scalpel!). 
Hartelius clearly has it right when she notes that procedural expertise is elevated in the context of Wikipedia. But I submit that this observation is true in each of the contexts that The Rhetoric of Expertise explores. Politically, historically, medically: in all arenas of life the careful search, assessment, analysis, deliberation about, and synthesis of information is supplanting "mere" discovery and distribution of information as the primary work of expertise.

This should not be terribly surprising. Thirty years ago, Jean-François Lyotard in The Postmodern Condition (1979/1984) predicted that the growth of information databases would transform social conceptions of knowledge from subject matter command to the procedural facilities that I am suggesting constitute networked expertise. As information becomes increasingly available, interlocutors lose the edge historically provided by knowledge differentials. Instead, advantage goes to those that can creatively connect the information that everyone has access to:

in games of perfect information, the best performativity cannot consist in obtaining ad-
ditional information ... it comes rather from arranging the data in a new way, which
is what constitutes a "move" properly speaking. This new arrangement is usually
achieved by connecting together series of data that were previously held to be inde-
pendent. This capacity to articulate what used to be separate can be called imagination.
Speed is one of its properties. It is possible to conceive the world of postmodern knowl-
edge as governed by a game of perfect information, in the sense that the data is in prin-
ciple accessible to any expert: there is no scientific secret. (Lyotard 1979/1984, 51-52)

It is certainly true that games of perfect information are probably impossible. Even with the increasing amount of information available, suggesting that any communicator would ever have something that could be characterized as perfect information on an issue like climate change stretches credulity. However, there is little doubt that access to data for citizens of networked societies is inching closer to the impossible ideal of "perfect information," since digital technologies enable new ways of collecting and synthesizing information and networked information architectures make it increasingly available.

As established information routines break down, simple possession of information will not itself give rhetorical advantage, since theoretically everyone will have access to that information (in theory only, of course, since access to information is articulated with various social identities like class). Interlocutors who can arrange an argument in a novel form will have more persuasive success. Here again, the blurry distinction between invention and disposition comes to the fore. Novel - that is to say imaginative or speedy - arrangements will garner more attention. This is certainly the case when Wikipedia becomes a clearinghouse for breaking news. Wikipedians produce an entry with external links that trace developments as soon as a major event, like a natural disaster or political gaffe, happens. Hartelius identifies this as the "rhetorical agility" of Wikipedia $(2011,155)$. Procedural expertise, like facility searching for information about unfolding events and synthesizing information into a cohesive narrative, becomes privileged in this environment. 
Procedural expertise is thus intimately linked to invention, but reframes its function. Invention is not about the "discovery" of some fact or claim, but about the ability to craft information in a way that generates a knowledge claim with widely perceived legitimacy. The focus on invention suggests that this is a more dramatic shift in how expertise functions in a networked era. It is certainly true that many subject matter experts author parts of articles, but their individual contributions are not inherently privileged. If another contributor is able to cobble together a compelling addition or emendation based on freely available and verifiable information from elsewhere on the internet, then the article may ultimately be refined. Thus, the ability to contribute to Wikipedia is not grounded in subject matter expertise per se, but in the ability to generate new ways of thinking about information in a way that is persuasive to other editors and/as readers.

This is not to say that subject-matter expertise is disappearing, or that all that is to know is known, or that pedagogy should be exclusively reoriented toward facility with search engines. Indeed, procedural expertise, the management and crafting of information, is relatively meaningless without a comprehensive database of topicspecific information from which skilled citizens can draw. Many-to-many communication, by filling databases with information on the scale needed to create more perfect information games, documents, organizes, and synthesizes massive amounts of information. This kind of integrative epistemic sociality, as Simon (2010) theorizes, is quite powerful in producing knowledge efficiently while embracing the cognitive diversity required in addressing complicated issues. The idea of epistemic sociality is instructive because it cues us to how collaborative invention works. In many ways, digital media have simply returned invention to its social roots after modernity's author-fetishizing interruption (LeFevre 1987; Pettitt 2007). Invention is a social act. Even acts of solo authorship are informed by prior experience with others and internal rhetorics modeled on debate (Billig 1987; Nienkamp 2001). It flourishes in dialogue between interlocutors, and blooms further when the dialogue becomes a many-to-many "polylogue" (Kerbrat-Orecchioni 2004; Marcoccia 2004). The sociality of invention is shown by how Wikipedia articles are crafted. Arguments that occur within the electronic infrastructure of Wikipedia shape the eventual prose of each entry. Wikipedians practice wordsmithing, fact-checking, and point-of-view negotiation. Each of these "procedural" expertises finds expression in an open-ended and ongoing collaboration that results in the (temporarily) finished article.

The inventional significance of many-to-many communication is not limited, however, simply to the production of discourse-it has the potential to alter the very agenda of public conversation. This is the second way that familiar information routines are disrupted by digital mediation. Experts in the modern era functioned as gatekeepers and, like all gatekeepers, had substantial power to shape the agenda for public conversation. This is certainly true for journalistic experts, but it is as true of other kinds of experts. Many-to-many communication in networked contexts, however, has: 
the potential to collapse the social and political spheres by creating forms of discourse outside the ambit of traditional politics and which deal with such matters as global justice, environmentalism, intimate relationships, different sexualities, frailty and personal risk, and which prioritize lay experience over professional expertise. (Bell et al. 2004, 117)

Some scholars speculate that bloggers, Wikipedians, and other digital intermediaries function essentially as "gatewatchers" or the "fifth estate" (Bruns 2005; Hayes 2008). When the doors - or floodgates - are opened to many-to-many communication, then the agenda-setting function of credentialed experts erodes.

This sphere collapse is not universally admired. Hartelius notes that "one of the most common accusations against Wikipedia today is that it elevates trivia to an unwarranted status," and, indeed, much Wikipedia content would be perceived by Encyclopaedia Britannica aficionados as trivia (2011, 141). Absent a strong, centralized agenda-setter, like an editor, the demarcation between "legitimate" and "trivial" topics loosens considerably. Wikipedians make entries for everything notable and let the attention market make judgments of relevance. ${ }^{2}$

This tension between legitimate and trivial topics can be traced at least as far back as Isocrates, who, in his Helen, disparaged orators that spoke on what he perceived as the lesser topics of salt and bumblebees. The boundaries between the legitimate and trivial, however, have eroded in tandem with the divide between the political and personal. When the many get involved in thematizing elements of public life on Wikipedia, they implicitly ask "Legitimate for who?" and "Trivial for who?" For example, Encyclopaedia Britannica, even the online version, has a paucity of entries on what used to be called subcultures and are now more favorably called counterpublics. The Encyclopaedia Britannica has no entry for "steampunk," a cultural counterpublic that blends the aesthetics of the 19th century with science fiction and fantasy elements. Wikipedia features a lengthy entry and several photographs taken by steampunk enthusiasts of home-made "steampunked" artifacts (like a desktop computer encased in an ornate, brass frame). The absence of a Wikipedia entry on something important to someone functions as an inventional siren, beckoning enterprising editors to author a serviceable article. People answer this inventional siren in order to contribute to knowledge production, engage in the pleasures of documentation, or perhaps just because it beats watching television.

As contributions to the massive encyclopedia project accumulate, Wikipedia editing may be seen as an extension of the documentary impulses that have become so prevalent in digital cultures. The cultural predilection for public sharing - witness reality television and social networking sites - is difficult to theorize apart from the ways in which digital media have made the preservation and circulation of information much easier than under analog conditions. For better or worse, the documentary drive is in part what fuels the collaborative invention of so many people on Wikipedia on issues that range in perceived importance. This is a genie that will seriously resist being returned to the bottle! Instead of simply moaning about the decline of civilization under the accumulated weight of triviality, scholars should examine how topical expansion 
invites new modes of informational filtering. Rather than the pre-publication filtering of an editor or other expert, who chooses what is relevant or trivial, many-to-many communication relies on a post-publication filtering model. The only expended resource is the volunteer time of the authors, so the opportunity costs involved in authoring an entire Wikipedia article devoted to tomacco (an obscure reference to an episode of The Simpsons) are small. The resource investment in that article is relatively minute and it might, some time far after publication, be made relevant in some situation.

If the first way that many-to-many communication reshapes the relationship between invention and expertise is to reshuffle traditional attention routines, the second significant effect of these new communication environments is a facilitation of multiperspectivalism. This multiperspectivalism emerges, not necessarily in the main article entry itself, but in the edit history and talk pages that constitute the substrata of Wikipedia. Herbert Gans $(1979 / 2004,2011)$ famously argued that traditional topdown news formats privilege particular views with the consequence that what gets covered is a very narrow slice of the actual news. How that news is framed shapes how citizens attend to it - if at all. Multiperspectival news, his proposed alternative, is journalism that draws in the opinions of the many in an attempt to better encompass available opinions. The resonance between Gans' assessment of top-down news versus multiperspectival news and the competing encyclopedias that are the subject of this essay is strong. Indeed, Axel Bruns (2006) has identified how digital media like independent websites, the blogosphere, and Wikipedia's news arm, Wikinews, actualize Gans' call for multiperspectival news. If multiperspectival news is desirable, then surely so is a multiperspectival encyclopedia. The many-to-many communication on the edit and talk pages reveals behind-the-scenes conflicts from multiple perspectives that need(ed) negotiation before some contingent consensus was reached. This kind of many-to-many multiperspectivalism introduces epistemic turbulence into encyclopedia knowledge production in a way that invites - even demands - a continual process of invention.

Publisher and author James Bridle recently gained international attention for a relatively simple stunt that demonstrates the relationship between Wikipedia and multiperspectivalism: he collected every change to the Wikipedia entry for "The Iraq War" between December 2004 and November 2009 and published them in 12 paper volumes (Bridle 2010): 12,000 changes; 7000 pages. As Bridle notes on his blog post describing the project:

It amounts to twelve volumes: the size of a single old-style encyclopaedia. It contains arguments over numbers, differences of opinion on relevance and political standpoints, and frequent moments when someone erases the whole thing and just writes "Saddam Hussein was a dickhead." (Bridle 2010)

Bridle's printing of the Iraq War entry is more performative than utilitarian, and what it performs is a shift from history to historiography. Noting this shift, Bridle (2010) writes: "This is what culture actually looks like: a process of argument, of dissenting 
and accreting opinion, of gradual and not always correct codification." The edit history of the Iraq War entry reveals the loci of disagreement: places where interlocutors simply cannot agree but also moments where the inventional process is required to develop common ground. The Iraq War entry is not just an entry about the conflict in Iraq; it is a documentation of the struggles, ideas, and feelings that constituted American and global public culture for an extended moment. Rhetorical historians couldand should - read these edit histories as archives.

Multiperspectivalism is functionally built into digital media because of the toggling action that hypertext invites. The edit history of the Iraq war entry exemplifies how Wikipedia oscillates between the "at" and "through" dynamic Richard Lanham $(1995,2006)$ theorizes as the interpretive strategy elicited by digital artifacts. According to Lanham, print "wants the gaze to remain THROUGH and unselfconscious all the time" $(1995,43)$. Readers of print look for the instrumental and purposive meaning of the text because of the standardization and aesthetic plainness of typography and the historical-cultural presumptions that print transparently reflects reality. By contrast, digital media involves movement between looking through the text for meaning and at the text itself for meaning. The hyperlink demonstrates this phenomenon: a hypertext reader can read through a sentence with hyperlinks for meaning, but they can also, by looking at the link, absorb more data: traces of prior discourse, the aesthetics of the typography, additional support for premises, corroborating evidence, primary material, and so on. The edit history of Wikipedia performs this reflexive function. One can read through the entry on any given topic, but then look at the entry's production by scrutinizing the edit history. A sophisticated reader can acquire a greater appreciation for the arguments and points of stasis that revolve around a particular entry. This is quite different from traditional engagements with subject matter experts, who often, as Hartelius puts it, use "finalizing extralinguistic means [such] as titles, degrees, and traditional credentials" to truncate conversation $(2010,146)$.

The at/through oscillation in Wikipedia introduces epistemic turbulence to encyclopedia knowledge. When there are accuracy or point of view concerns in a Wikipedia article, some variant of "this article is under dispute" often appears at the top of controversial articles, warning viewers that differences of opinion have prevented consensus and thus flagging epistemic turbulence. "This article is under dispute" activates an inventional process to reconcile or otherwise negotiate competing versions of the article. Sometimes editors facilitate this delicately by "teaching the controversy" within the body of the entry itself. Other times, the dispute is serious enough to activate other kinds of intervention that require the invention of arguments to make one's case that the article should read one way instead of another. The community page on "Dispute Resolution" identifies norms to avoid conflict (focusing on content, staying cool, discussing with other party) and methods to resolve conflict (editorial assistance, third-party opinion, informal mediation, formal mediation and, if all else fails, arbitration). These methods to encourage reflexivity are sometimes at odds with the passion that Wikipedia authors bring to their entries, yet they are prob- 
ably desirable in as much as one cannot simply embrace an "anything goes" kind of relativistic multiperspectivalism.

A system that relies on the articulation, negotiation, and blending of multiple perspectives must lean very heavily on reflexivity, what Hartelius refers to as the "network trust" embedded in Wikipedia, in order to create coherent entries perceived as legitimate by the contributing editors. But it also must encourage people to participate in the first place. Since Wikipedia is an ongoing, aggregative project, the community pages that identify the norms of editing practice animate participatory expertise. As Hartelius notes: "Wikipedia's invitational rhetoric-urging interactivity that lets all users edit each other-ultimately prioritizes dialogue over policy" $(2011,513)$. After all, as one of Wikipedia's introductory pages notes: "You can't break Wikipedia. Anything can be fixed or improved later" (Wikipedia n.d. c). Although Hartelius suggests that this signifies an emergence of a kind of dialogical expertise, I would, in the spirit of this essay, refer to it as polylogical expertise. In order for polylogues to thrive, a core edict of Wikipedia - "Don't be afraid to edit" - is required for the many to get involved. The open source ethos - for software or encyclopedia production demands an experimental, even playful, spirit of invention.

\section{Conclusion}

Hartelius' (2011) The Rhetoric of Expertise will sustain a variety of conversations about the nature and role of expertise for years to come. The tensions that she identifies as constituting two different conceptualizations of expertise represented by the Encyclopaedia Britannica and Wikipedia are representative of the tensions to be found in the transition from a one-to-many broadcast model of expertise to a participatory model of expertise reliant on many-to-many communication. This shift, I argued, can be further appreciated by examining how many-to-many communication impacts invention along two lines: reshuffling established information routines and creating epistemic turbulence through multiperspectivalism. To be sure, one effort to interpret how expertise is reconfigured in an internetworked era, like my own or Hartelius' The Rhetoric of Expertise, is not enough: only the many can, in the collaborative intercast of discussion, extend and contest these observations.

Acknowledgements - The author would like to thank Scott Church, Carly S. Woods, Johanna Hartelius, and Jim Collier for their perceptive contributions to this essay.

\section{Notes}

[1] Although Wikipedia officially forbids "original research," I employ the term "knowledge production" to refer to the social practices that go in to the crafting of each article. Wikipedia articles produce knowledge by drawing upon original and sourced research, synthesizing it, and then updating it over time. Although not based on "original research" as the academy under- 
stands the term, we ought not foreclose the possibility that these articles do in fact produce knowledge for some social actors.

[2] In contrast to the Encyclopaedia Britannica, which makes judgments of legitimacy and triviality based on perceived importance, Wikipedia adjudicates these distinctions based on received attention. Articles must meet Wikipedia's notability standard, which is "an attempt to assess whether the topic has received 'attention from the world at large'" through secondary sources (Wikipedia n.d. a). While articles can be made on virtually any topic, there is an ongoing process to delete or merge entries that in some way fail Wikipedia's notability criterion (Wikipedia n.d. d).

\section{References}

Bell, D., B. Loader, N. Pleace, and D. Schuler. 2004. Cyberculture: The Key Concepts. New York: Routledge.

Billig, M. 1987. Arguing and Thinking: A Rhetorical Approach to Social Psychology. Cambridge: Cambridge University Press.

Brandt, K. R., L. M. Cooper, and S. A. Dewhurst. 2005. Expertise and recollective experience: Recognition and memory for familiar and unfamiliar academic subjects. Applied Cognitive Psychology 19 (9): 1113-25.

Bridle, J. 2010. On Wikipedia, cultural patrimony, and historiography. Booktwo.org [referenced June 13, 2011]. Available from http://booktwo.org/notebook/wikipedia-historiography/

Bruns, A. 2005. Gatewatching: Collaborative Online News Production. New York: Peter Lang.

Bruns, A. 2006. Wikinews: The next generation of alternative online news? SCAN Journal 3 (3) [referenced June 13, 2011].

Available from http://scan.net.au/scan/journal/print.php?j_id=9\&journal_id=69

Carey, J. 2005. Historical pragmatism and the internet. New Media and Society 7 (4): 443-55.

Dewar, J. A., and P. H. Ang. 2007. The cultural consequences of printing and the internet. In Agent of Change: Print Culture Studies after Elizabeth L. Eisenstein, edited by S. A. Baron, E. N. Lindquist, and E. F. Shevlin, pp. 365-77. Amherst: University of Massachusetts Press.

Eisenstein, E. 1979. The Printing Press as an Agent of Change: Communications and Cultural Transformations in Early Modern Europe. Cambridge: Cambridge University Press.

Fuller, S. 2006. The constitutively social character of expertise. In The Philosophy of Expertise, edited by E. Selinger and R. P. Crease, pp. 342-57. New York: Columbia University Press.

Gans, H. 1979/2004. Deciding What's News: A Study of CBS Evening News, NBC Nightly News, Newsweek, and Time. Evanston, IL: Northwestern University Press.

Gans, H. 2011. Multiperspectival news revisited: Journalism and representative democracy. Journalism 12 (1): 3-13.

Halavais, A. 2009. Search Engine Society. Cambridge: Polity Press.

Hartelius, J. 2010. Wikipedia and the emergence of dialogical expertise. Southern Communication Journal 75 (5): 505-26.

Hartelius, J. 2011. The Rhetoric of Expertise. Lanham, MD: Lexington Books.

Hayes, A. S. 2008. Press Critics are the Fifth Estate: Media Watchdogs in America. Westport, CT: Praeger. 
Johnson, D. 2003. Campaign politics, the internet, and ethics. In The Civic Web: Online Politics and Democratic Values, edited by D. Anderson and M. Cornfeld, pp. 9-19. Lanham, MD: Rowman \& Littlefield.

Kerbrat-Orecchioni, C. 2004. Introducing polylogue. Journal of Pragmatics 36 (1): 1-24.

Lanham, R. 1995. The Electronic Word: Democracy, Technology, and the Arts. Chicago: University of Chicago Press.

Lanham, R. 2006. The Economics of Attention: Style and Substance in the Information Age. Chicago: University of Chicago Press.

LeFevre, K. B. 1987. Invention as a Social Act. Carbondale, IL: Southern Illinois Press.

Lyotard, J. 1979. The Postmodern Condition: A Report on Knowledge. Minneapolis: University of Minnesota Press, 1984.

Marcoccia, M. 2004. On-line polylogues: Conversation structure and participation framework in internet newsgroups. Journal of Pragmatics 36 (1): 115-45.

Nienkamp, J. 2001. Internal Rhetorics: Toward a History and Theory of Self-Persuasion. Carbondale, IL: Southern Illinois Press.

Pettitt, T. 2007. Before the Gutenberg parenthesis: Elizabethan-American compatibilities. Paper presented at MIT5: Creativity, ownership, and collaboration in the digital age [referenced June 13, 2011].

Available from http://web.mit.edu/comm-forum/mit5/papers/pettitt_plenary_gutenberg.pdf

Sanger, L. 2009. The fate of expertise after Wikipedia. Episteme 6 (1): 52-73.

Simon, J. A socio-epistemological framework for scientific publishing. Social Epistemology 24 (3): 201-18.

Stalder, F. 2006. Manuel Castells: The Theory of the Network Society. Cambridge: Polity Press.

Walsh, P. 2003. That withered paradigm: The web, the expert, and the information hegemony. In Democracy and New Media, edited by H. Jenkins and D. Thorburn, pp. 365-72. Cambridge, MA: MIT Press.

Wikipedia. n.d. a. Notability in Wikipedia [referenced March 23, 2011]. Available at http://en.wikipedia.org/wiki/Notability in Wikipedia

Wikipedia. n.d. b. Reliability of Wikipedia [referenced March 23, 2011]. Available at: http://en.wikipedia.org/wiki/Reliability_of_Wikipedia

Wikipedia. n.d. c. What is Wikipedia? [referenced February 25, 2011]. Available at: http://en.wikipedia.org/wiki/Wikipedia:Introduction

Wikipedia. n.d. d. Wikipedia: Articles for deletion [referenced March 23, 2011]. Available at: http://en.wikipedia.org/wiki/Wikipedia:Articles_for_deletion 